\title{
A systematic study of relativistic (e, 2e) collisions in comparison with experiment
}

\author{
D H Jakubassa-Amundsen \\ Physics Section, University of Munich, D-8046 Garching, Federal Republic of Germany
}

Received 28 November 1991

\begin{abstract}
Triply-differential cross sections for K-shell ionization by fast electron impact are calculated within the first-order Coulomb Born approximation for the case of a coplanar symmetric geometry. Comparison is made with experimental data on $300 \mathrm{keV}$ and $500 \mathrm{keV} \mathrm{e}^{-}+\mathrm{Cu}, \mathrm{Ag}$ and $\mathrm{Au}$. For the two lighter targets, the binary peak region is reasonably well described by a theory which uses semirelativistic electronic eigenfunctions to the target field, provided spin-flip is included. For the gold target, the Coulomb Born approximation seriously overestimates the data, pointing to the necessity of a fully relativistic description of the electronic states.
\end{abstract}

Triply-differential cross sections for inner-shell ionization in electron-atom collisions provide a sensitive test for theoretical models since a coincident detection of the two momentum-analysed outgoing electrons allows for a complete determination of the collision kinematics (McCarthy and Weigold 1976, Ehrhardt et al 1986, LahmamBennani 1991). For fast collisions where a first-order treatment of the electron-electron interaction should be appropriate and polarization effects may be neglected, the basic information to be extracted from $(e, 2 e)$ cross sections concerns thus the particulars of the electronic wavefunctions.

For low-energy electron scattering it has become standard to use a Hartree-Focktype function for the bound target electron, and numerically generated scattering states

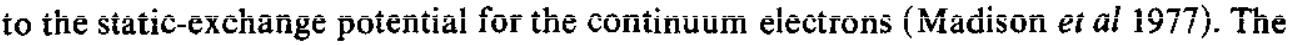
multiple partial wave expansion required for the evaluation of the cross section becomes, however, prohibitive at high projectile energies, and such elaborate wavefunctions have up to now only been employed for energies of $150 \mathrm{keV}$ and below (Pindzola and Buie 1988).

The (e, 2e) experiments have recently been extended to the relativistic regime with impact energies up to $500 \mathrm{keV}$ (Schüle and Nakel 1982, Ruoff and Nakel 1987, Bonfert et al 1991, Walters et al 1991). Apart from the distorted-wave Born approximation of Pindzola and Buie (1988), the theoretical approaches for relativistic electron impact ionization are restricted to the plane-wave Born approximation (PWBA; Möller 1932), where the primary electron is described by Dirac plane waves, while the target field is accounted for in the states of the secondary electron (Das 1972, Davidovic et al 1978). Such a simple piescription works well for weak target fields and for asymmetric energy sharing between the two outgoing electrons (the secondary electron being much slower than the primary one). However, it has been shown that the plane-wave Born approximation overestimates experiments with a symmetric energy sharing, the more so, the heavier the target nucleus (Bonfert et al 1991). Therefore we have developed a theory, termed Coulomb Born approximation, which comprises the advantages of the PWBA and the distorted-wave theory: The target potential is considered in the states 
of the primary electron, but by choosing non-relativistic Coulomb waves multiplied by a Dirac spinor for all unbound electronic states, a partial wave expansion can be avoided (Jakubassa-Amundsen 1989).

Adopting the argumentation of Moiseiwitsch (1980) that spin-flip contributes little to total ionization cross sections at moderate relativistic impact energies, we had neglected spin-flip in our previous work. However, it has recently been shown by Walters et al (1991) within a PWBA treatment, that inclusion of spin-flip leads to a considerable enhancement of $(e, 2 e)$ cross sections for a symmetric energy sharing where the momenta of both outgoing electrons are large. In the present work, we therefore allow for spin-flip, but we also test the wavefunction effects by (i) replacing the Darwin $K$-shell function by a relativistic hydrogenic function, (ii) replacing the non-relativistic Coulomb wave for the secondary electron by a Darwin function (in our PWBA code), and (iii) accounting for the final-state interaction by a simple modification of the two-electron wavefunction. The importance of the non-perturbative treatment of the electron-electron interaction had been pointed out by Brauner et al (1989) for small relative momenta of the outgoing electrons.

A detailed description of the Coulomb Born theory has been given earlier (Jakubassa-Amundsen 1989). In short, the triply-differential cross section for ejecting a (secondary) electron from the target subshell $i$ with occupation number $N_{i}$ into the solid angle $d \Omega_{\kappa f}$, while scattering the impinging (primary) electron into the solid angle $\mathrm{d} \Omega_{k_{f}}$, is given by (in atomic units, $\hbar=m=e=1$ )

$\frac{\mathrm{d}^{3} \sigma}{\mathrm{d} E_{\kappa_{f}} \mathrm{~d} \Omega_{\kappa_{f}} \mathrm{~d} \Omega_{k_{f}}}=\frac{N_{i}}{c^{6} k_{i}} \kappa_{f} E_{\kappa_{f}} k_{f} E_{k_{f}} E_{k_{i}} \sum_{s_{f} \sigma_{f} s_{i} \sigma_{i}}\left|W_{s_{f} \sigma_{f} s_{i} \sigma_{f}}^{\mathrm{d}}\left(\boldsymbol{k}_{f}, \boldsymbol{\kappa}_{f}\right)-W_{s_{f} \sigma_{f} s_{i} \sigma_{t}}^{\mathrm{ex}}\left(\boldsymbol{k}_{f}, \boldsymbol{\kappa}_{f}\right)\right|^{2}$

where $i$ characterizes the initial and $f$ the final state. The momenta and total energies of the primary and the secondary electron are denoted by $\boldsymbol{k}, \boldsymbol{\kappa}$ and $E_{k}, E_{\kappa}$, respectively, while their spin quantum numbers are termed $s$ and $\sigma$, respectively. Spin-flip is included by summing over all 16 combinations of the four spins $(s, \sigma=\{+,-\})$. The exchange interaction is accounted for by subtracting from the direct term, $W^{d}$, an exchange term, $W^{\text {ex }}$, which is obtained from the direct term by exchanging momenta and spins of the two outgoing electrons according to $W_{s_{f} \sigma_{f} s_{i} \sigma_{i}}^{\text {ex }}\left(\boldsymbol{k}_{f}, \boldsymbol{\kappa}_{f}\right)=W_{\sigma_{f} s_{f} s_{i} \sigma_{l}}^{\mathrm{d}}\left(\boldsymbol{\kappa}_{f}, \boldsymbol{k}_{f}\right)$. Since the wavefunctions of the primary and the secondary electron, $\psi_{f}^{\left(s_{f}\right)}$ and $\phi_{f}^{\left(\sigma_{f}\right)}$, have the same structure, being composed of a Coulomb wave $\psi_{k_{f}}, \psi_{\kappa_{f}}$ times a Dirac spinor $a_{k_{f}}^{\left(s_{f}\right)}$, $a_{\kappa j}^{\left(\sigma_{j}\right)}$ (times a normalization constant; for their definition see e.g. Bjorken and Drell 1964), respectively, the so obtained exchange term is-within the Coulomb Born approximation-_exact. Taking for the bound-state function $\phi_{i}^{\left(\sigma_{i}\right)}$ a Darwin function, i.e. a spinor $a_{i}^{\left(\sigma_{i}\right)}$ acting on a hydrogenic 1 s state, $\psi_{i}$, (where $a_{i}^{\left(\sigma_{i}\right)}$ is obtained from $a_{k_{i}}^{\left(\sigma_{i}\right)}$ by replacing $k_{i \lambda}$ by $-\mathrm{i} \partial_{\lambda}(\lambda=z, \pm)$ where $k_{ \pm}=k_{x} \pm \mathrm{i} k_{y}$ and $\left.\partial_{ \pm}=\partial / \partial_{x} \pm \mathrm{i} \partial / \partial_{y}\right)$, the direct term which accounts for the electron-electron coupling to first order, is obtained from

$$
\begin{aligned}
& W_{s f}^{\mathrm{d}} \sigma_{f} s_{i} r_{i}\left(\boldsymbol{k}_{f}, \boldsymbol{\kappa}_{f}\right) \\
& =C_{f i} \int \frac{\mathrm{d} \boldsymbol{q}}{q^{2}-q_{0}^{2}-\mathrm{i} \varepsilon}\left[\left(a_{k_{f}}^{\left(s_{f}\right)+} a_{k_{i}}^{\left(s_{i}\right)}\right)\left\langle\psi_{k_{f}}\left|\mathrm{e}^{-\mathrm{iqq}}\right| \psi_{k_{i}}\right\rangle\left\langle\psi_{\kappa_{f}}\left|\mathrm{e}^{\mathrm{i} q \sigma}\left(a_{\kappa_{j}}^{\left(\sigma_{j}\right)+} a_{i}^{\left(\sigma_{i}\right)}\right)\right| \psi_{i}\right\rangle\right. \\
& \left.-\left(a_{k_{f}}^{\left(s_{f}\right)+} \boldsymbol{\alpha} a_{k_{i}}^{\left(s_{j}\right)}\right\rangle\left\langle\psi_{k_{f}}\left|\mathrm{e}^{-\mathrm{i} \boldsymbol{q} r}\right| \psi_{k_{\mathrm{j}}}\right\rangle\left\langle\psi_{\kappa_{f}}\left|\mathrm{e}^{\mathrm{i} \boldsymbol{i} r}\left(a_{\kappa_{f}}^{\left(\sigma_{f}\right)+} \boldsymbol{\alpha} a_{i}^{\left(\sigma_{i}\right)}\right)\right| \psi_{i}\right\rangle\right] \\
& C_{f i}=\left(\frac{E_{k_{i}}+m c^{2}}{2 E_{k_{i}}} \frac{E_{k_{f}}+m c^{2}}{2 E_{d_{f}}} \frac{E_{\kappa_{f}}+m c^{2}}{2 E_{\kappa_{f}}} \frac{1}{1+\left[Z_{\mathrm{T}} c /\left(E_{i}+m c^{2}\right)\right]^{2}}\right)^{1 / 2} \quad \varepsilon=+0
\end{aligned}
$$


where $E_{i}$ is the total bound-state energy, $Z_{T}$ is the target charge and the matrices $\boldsymbol{\alpha}=\left(\alpha_{x}, \alpha_{y}, \alpha_{z}\right)$ are the (magnetic) Dirac matrices (Bjorken and Drell 1964). The fourth component of the momentum transfer $\left(q, q_{0}\right)$ is $q_{0}=\left(E_{k_{i}}-E_{k_{f}}\right) / c$, and $\boldsymbol{r}$ is the coordinate of the respective electron in the target reference frame. From equation (2), the planewave Born approximation is easily obtained by replacing $\psi_{k_{i}}$ and $\psi_{k_{f}}$ by plane waves. Hence, $\left\langle\psi_{k_{f}}|\exp (-\mathrm{i} q r)| \psi_{k_{i}}\right\rangle=\delta\left(\boldsymbol{q}+\boldsymbol{k}_{f}-\boldsymbol{k}_{i}\right)$ such that the momentum transfer is fixed, $\boldsymbol{q}=\boldsymbol{k}_{i}-\boldsymbol{k}_{f}$.

In the following we restrict ourselves to a coplanar symmetric geometry where both electrons are ejected in-plane with the impinging electron, such that $k_{f}=\kappa_{f}, \vartheta_{k_{f}}=\vartheta_{\kappa_{f}}$ and $\varphi_{k_{f}}-\varphi_{\kappa_{f}}=\pi$ (where $k, \vartheta_{k}, \varphi_{k}$ are the spherical coordinates of $k$ and the $z$ axis is taken along $\left.\boldsymbol{k}_{i}\right)$. Since in this case one has the symmetry property, $W_{s_{f} \sigma_{f} s_{i} \sigma_{l}}^{\mathrm{d}}\left(\boldsymbol{k}_{f}, \boldsymbol{\kappa}_{f}\right)=$ $\dot{W}_{s_{f} \sigma_{f} s_{i} \sigma_{i}}^{\mathrm{d}}\left(\boldsymbol{\kappa}_{f}, \boldsymbol{k}_{f}\right)$, all terms with equal final spin quantum numbers, $s_{f}=\sigma_{f}$, do not contribute to the spin sum in (1). Defining $\hat{W}_{s_{f} \sigma_{f} s_{i} \sigma_{i}} \equiv W_{s_{f} \sigma_{f} s_{i} \sigma_{i}}^{\mathrm{d}}\left(\boldsymbol{k}_{f}, \boldsymbol{\kappa}_{f}\right) / C_{f i}$, one is left with a sum of eight terms which are pairwise identical

$$
\begin{aligned}
\frac{\mathrm{d}^{3} \sigma}{\mathrm{d} E_{\kappa_{f}} \mathrm{~d} \Omega_{\kappa_{f}} \mathrm{~d} \Omega_{k_{f}}} & \\
= & \frac{2 N_{i}}{8 c^{6} k_{i}} \kappa_{f} k_{f} \Delta E_{k_{i}} \Delta E_{k_{f}} \Delta E_{\kappa_{f}} \frac{1}{1+\left(Z_{\mathrm{T}} c / \Delta E_{i}\right)^{2}} \\
& \times\left[\left|\hat{W}_{+-+-}-\hat{W}_{-+++}\right|^{2}+\left|\hat{W}_{-+-+}-\hat{W}_{+--+}\right|^{2}\right. \\
& \left.+\left|\hat{W}_{+-++}-\hat{W}_{-+++}\right|^{2}+\left|\hat{W}_{-+--}-\hat{W}_{+---}\right|^{2}\right]
\end{aligned}
$$

where $\Delta E_{p} \equiv E_{p}+m c^{2}$ with $p=i, k_{i}, k_{f}$ and $\kappa_{f}$. For the evaluation of (2), four integrals have to be calculated numerically with the techniques described in the earlier paper

$$
I_{0}^{\mathrm{d}}=\int \frac{\mathrm{d} \boldsymbol{q}}{q^{2}-q_{0}^{2}-\mathrm{i} \varepsilon}\left\langle\psi_{k_{f}}\left|\mathrm{e}^{-\mathrm{i} \boldsymbol{q} \cdot r}\right| \psi_{k_{i}}\right\rangle\left\langle\left.\psi_{\kappa_{f}}\left|\mathrm{e}^{\mathrm{i} \boldsymbol{q} \cdot r}\right|\right|_{\partial_{\lambda} \psi_{i}} ^{\psi_{i}}\right\rangle \quad \lambda=z, \pm
$$

where $I_{0}^{\mathrm{d}}$ and $I_{\lambda}^{\mathrm{d}}$ refer to $\psi_{i}$ and $\partial_{\lambda} \psi_{i}$, respectively. With the help of the definitions $\beta_{0} \equiv 1+c^{2} k_{f z} k_{i} /\left(\Delta E_{k_{f}} \Delta E_{k_{i}}\right), \quad \gamma_{0} \equiv c^{2} k_{i} /\left(\Delta E_{k_{f}} \Delta E_{k_{i}} \Delta E_{\kappa_{f}}\right), \quad \gamma_{i} \equiv \mathrm{i} c^{2} / \Delta E_{i}$ and $\delta_{i} \equiv$ $\gamma_{i}\left(\beta_{0} \kappa_{f z} / \Delta E_{\kappa_{f}}-k_{i} / \Delta E_{k_{1}}-k_{f z} / \Delta E_{k f}\right)$, the contributions to (3) take the following form

$$
\begin{aligned}
& \hat{W}_{+-+-}=I_{0}^{\mathrm{d}}\left(\beta_{0}-\frac{c^{2} \kappa_{f z}}{\Delta E_{\kappa_{f}}}\left(\frac{k_{i}}{\Delta E_{k_{i}}}+\frac{k_{f z}}{\Delta E_{k_{f}}}\right)-\frac{2 c^{2} \kappa_{f \pm} k_{f \mp}}{\Delta E_{k_{f}} \Delta E_{\kappa_{f}}}\right)-I_{z}^{\mathrm{d}} \delta_{i}-I_{\mp}^{\mathrm{d}} \gamma_{i} \beta_{0} \frac{\kappa_{f \pm}}{\Delta E_{\kappa_{f}}} \\
& \hat{W}_{+-++}=\mp I_{0}^{\mathrm{d}} \frac{c^{2} \kappa_{f \pm}}{\Delta E_{\kappa_{f}}}\left(\frac{k_{i}}{\Delta E_{k_{i}}}+\frac{k_{f z}}{\Delta E_{k_{f}}}\right) \mp I_{z}^{\mathrm{d}} \gamma_{i} \beta_{0} \frac{\kappa_{f \pm}}{\Delta E_{\kappa_{f}}} \pm I_{ \pm}^{\mathrm{d}} \delta_{i} \\
& \hat{W}_{++++}= \pm I_{0}^{\mathrm{d}} \frac{c^{2} k_{f \pm}}{\Delta E_{k_{f}}}\left(\frac{k_{i}}{\Delta E_{k_{i}}}-\frac{\kappa_{f z}}{\Delta E_{\kappa_{f}}}\right) \mp I_{z}^{\mathrm{d}} \gamma_{i}\left(\gamma_{0} k_{f \pm} \kappa_{f z}-\frac{k_{f \pm}}{\Delta E_{k_{f}}}\right) \\
& \mp I_{ \pm}^{\mathrm{d}} \gamma_{i}\left(\gamma_{0} k_{f \pm} \kappa_{f \mp}-\frac{2 k_{i}}{\Delta E_{k_{i}}}+\frac{2 k_{f z}}{\Delta E_{k_{f}}}\right) \\
& \hat{W}_{-++-}=-I_{0}^{\mathrm{d}}\left[\frac{2 c^{2} \kappa_{f z}}{\Delta E_{\kappa_{f}}}\left(\frac{k_{i}}{\Delta E_{k_{1}}}-\frac{k_{f z}}{\Delta E_{k_{f}}}\right)-\frac{c^{2} k_{f \pm} \kappa_{f \mp}}{\Delta E_{k_{f}} \Delta E_{\kappa_{f}}}\right]+I_{z}^{\mathrm{d}} \gamma_{i}\left(\gamma_{0} k_{f \pm} \kappa_{f \mp}-\frac{2 k_{f}}{\Delta E_{k_{i}}}+\frac{2 k_{f z}}{\Delta E_{k_{f}}}\right) \\
& -I_{\mp}^{\mathrm{d}} \gamma_{i}\left(\gamma_{0} k_{f \pm} \kappa_{f z}-\frac{k_{f \pm}}{\Delta E_{k_{f}}}\right)
\end{aligned}
$$


where the upper and lower signs correspond to the upper and lower spin combination of $\hat{W}_{s_{f} \sigma_{f} s_{i} \sigma_{i}}$, respectively.

The triply-differential cross sections for the $\mathrm{K}$-shell ionization of $\mathrm{Cu}, \mathrm{Ag}$ and $\mathrm{Au}$ by $300 \mathrm{keV}$ and $500 \mathrm{keV}$ electrons as a function of the emission angle are shown in figure 1. The energy of the outgoing electrons is fixed by energy conservation, $E_{k_{f}}=E_{\kappa_{f}}=$ $\left(E_{k_{i}}+E_{i}\right) / 2$. In the data from Nakel and his group (Bonfert et al 1991, Walters et al 1991) the binary peak around $\vartheta_{\kappa_{f}}=40^{\circ}$ is clearly visible for the lighter targets. It appears when the momentum transfer $q$ matches the momentum $\kappa_{f}$ of the secondary electron, provided the ratio $Z_{\mathrm{T}} / k_{i}$ is sufficiently small. This means that only small intrinsic momenta of the bound-state electron are required, the peak shape being determined by the momentum distribution of the bound state.

Comparison is made with the Coulomb Born theory (using Slater-screened wavefunctions and experimental binding energies) with and without the inclusion of spin-filp. It is found that consideration of spin-nfip gives a significant enhancement of the cross sections, leading to an improved agreement with the experimental data. The only exception concerns the heaviest $\mathrm{Au}$ ) target with large discrepancies between theory and experiment.

Plane-wave Born results with the same choice of wavefunctions $\phi_{i}^{\left(\sigma_{i}\right)}$ and $\phi_{f}^{\left(\sigma_{f}\right)}$ and the same prescription for the exchange term (which, however, is no longer exact) are included in figure 1. As has been shown by Walters et al (1991), these results lead to a considerable overprediction of the experimental data for the heavier targets, the more so, when spin-flip is included. However, due to the similar structure of the two theories, the spin-flip enhancement is very much the same as that found in the Coulomb Born theory. Quantifying this enhancement by the ratio $R=\mathrm{d}^{3} \sigma(f l i p) / \mathrm{d}^{3} \sigma$ (non-flip) of the cross sections with and without consideration of spin-flip, we have found a difference between the PWBA and the Coulomb Born results for $R$ of at most $10 \%$. Also, $R$ is rather insensitive to the choice of the target species $\left(R_{\mathrm{Cu}}, R_{\mathrm{Ag}}\right.$ and $R_{\mathrm{Au}}$ differ from each other by less than $10 \%$ ). However, the spin-flip enhancement depends strongly on angle and on the collision energy. The increase of $R$ with $E_{k_{i}}$ is shown in figure 2 for a $\mathrm{Cu}$ target within the PWBA. $R$ reaches a factor of 2 in the binary peak maximum at a kinetic energy $E_{k_{i}}-m c^{2} \sim 1 \mathrm{MeV}$, and spin-flip even is the dominant mechanism at the higher energies. Since the momentum transfer $\boldsymbol{q}=\boldsymbol{k}_{i}-\boldsymbol{k}_{f}$ increases (for fixed $E_{k_{s}}$ ) with angle, so does $R$. At ultrarelativistic energies, the focusing of the two electrons into the forward direction leads, however, to the largest spin-flip enhancement at small angles.

Making use of the similarities between the Coulomb Born and the plane-wave Born approximation which led to the correct estimate of the spin-flip enhancement in the pwBA, we apply in the following this much simpler theory for the investigation of wavefunction effects. First of all, we have studied the importance of describing the initial target state by an improved wavefunction. To this aim, we have replaced the Darwin function by a hydrogenic relativistic function

$$
\begin{array}{cc}
\phi_{i}^{\left(\sigma_{i}\right)}(r)=u_{i}^{\left(\sigma_{1}\right)} \varphi_{i}(r) & \varphi_{i}(r)=\frac{1}{\sqrt{\pi}} Z_{\mathrm{T}}^{3 / 2} \mathrm{e}^{-Z_{\mathrm{T}} r}\left(2 Z_{\mathrm{T}} r\right)^{\gamma-1} \frac{1}{\sqrt{\Gamma(2 \gamma+1)}} \\
u_{i}^{(+)}=\left(\begin{array}{c}
\sqrt{1+\gamma} \\
0 \\
\mathrm{i} \sqrt{1-\gamma} \cos \theta \\
\mathrm{i} \sqrt{1-\gamma} \sin \theta \mathrm{e}^{\mathrm{i} \varphi}
\end{array}\right) \quad u_{i}^{(-)}=\left(\begin{array}{c}
0 \\
\sqrt{1+\gamma} \\
\mathrm{i} \sqrt{1-\gamma} \sin \theta \mathrm{e}^{-\mathrm{i} \varphi} \\
-\mathrm{i} \sqrt{1-\gamma} \cos \theta
\end{array}\right)
\end{array}
$$



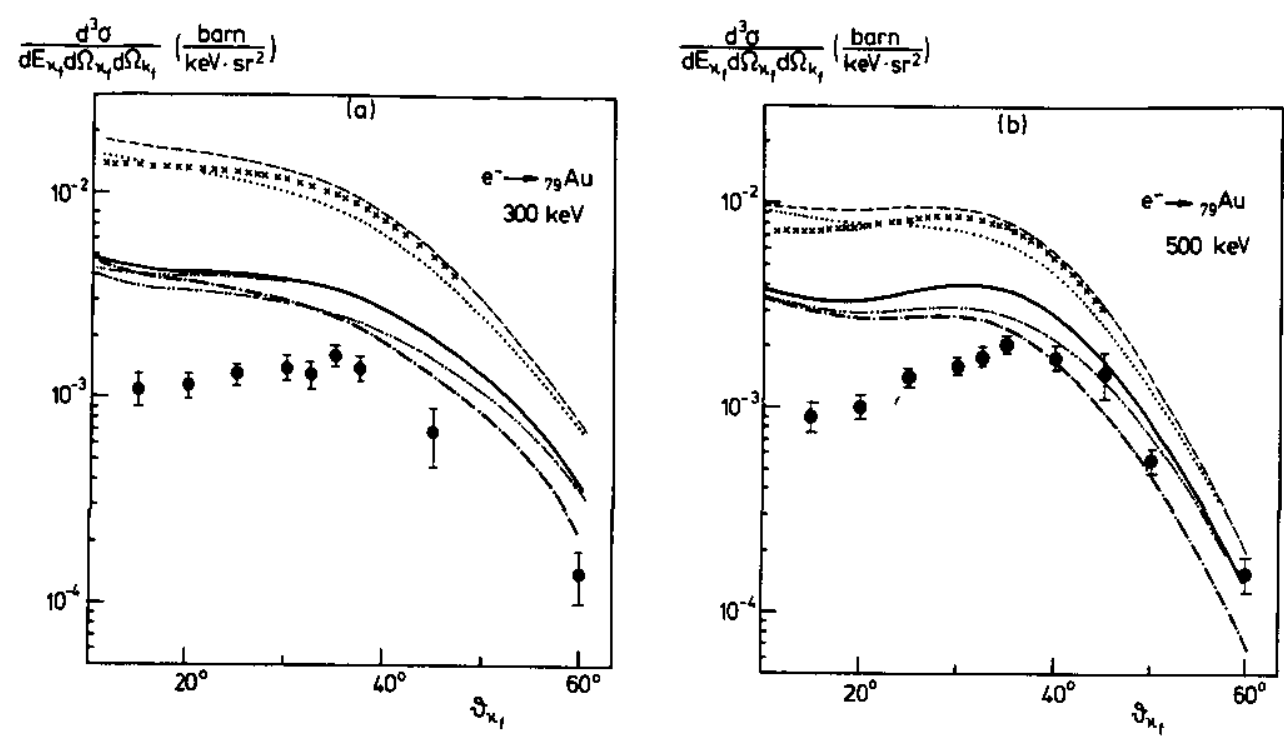

$\frac{d^{3} \sigma}{d E_{x p} d \Omega_{x f} d \Omega_{k_{f}}}\left(\frac{\text { barn }}{k e V \cdot s r^{2}}\right)$
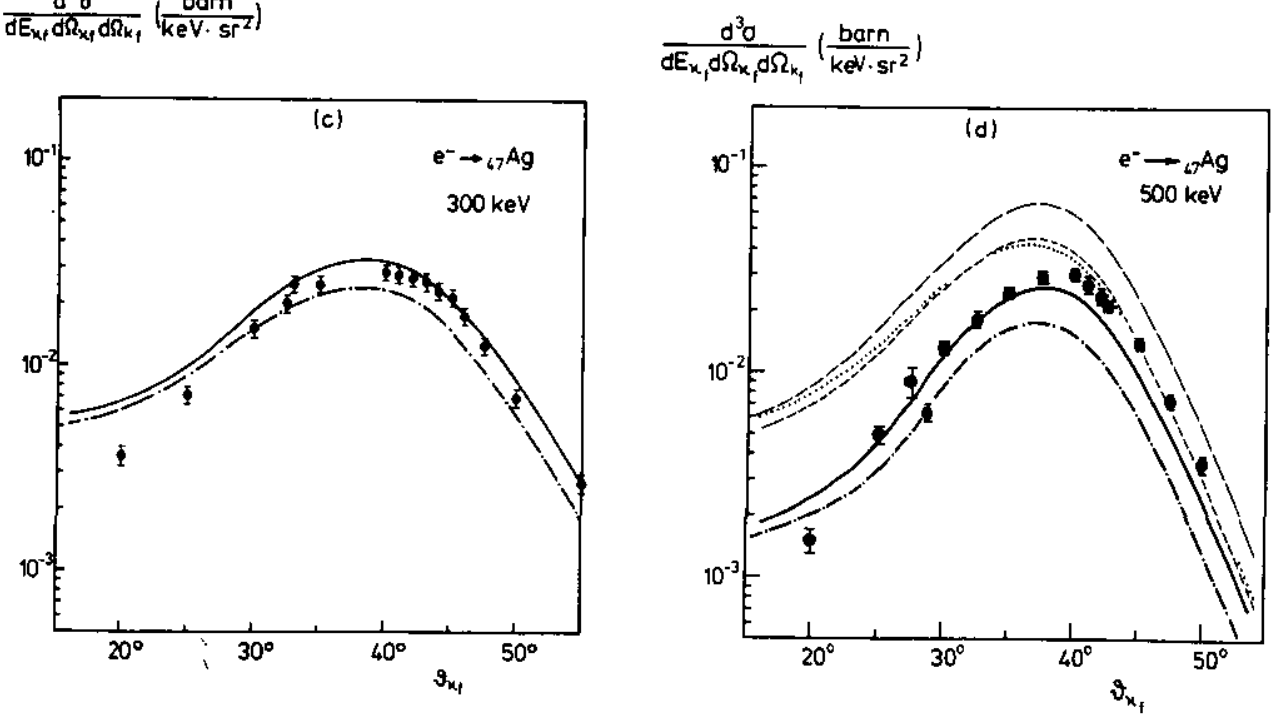

Figure 1. Triply-differential cross sections for $\mathrm{K}$-shell ionization of $\mathrm{Au}(a),(b), \mathrm{Ag}(c)$, $(d)$ and $\mathrm{Cu}(e),(f)$ by $300 \mathrm{keV}$ and $500 \mathrm{keV}$ electrons in coplanar symmetric geometry as a function of emission angle $\vartheta_{\kappa_{f}}$. Shown are the data $(\bar{\phi})$ from Bonfert et al (1991) and Walters $e t$ al (1991) in comparison with theory: Coulomb Born theory with spin-flip (—) and without spin-flip (-.-), plane-wave Born theory with (--) and without (---) spin-flip, all theories with a Darwin K-shell function. Also shown is the PWBA without spin-flip, using a Dirac K-shell function $(\cdots)$, and using Darwin functions for the bound and free secondary electron $(x \times x)$, as well as the spin-fip Coulomb Born theory with the correction factor $F_{i}(-\cdots-)$ and with the correction factor $F_{\text {ee }}(-\cdots-)$. 

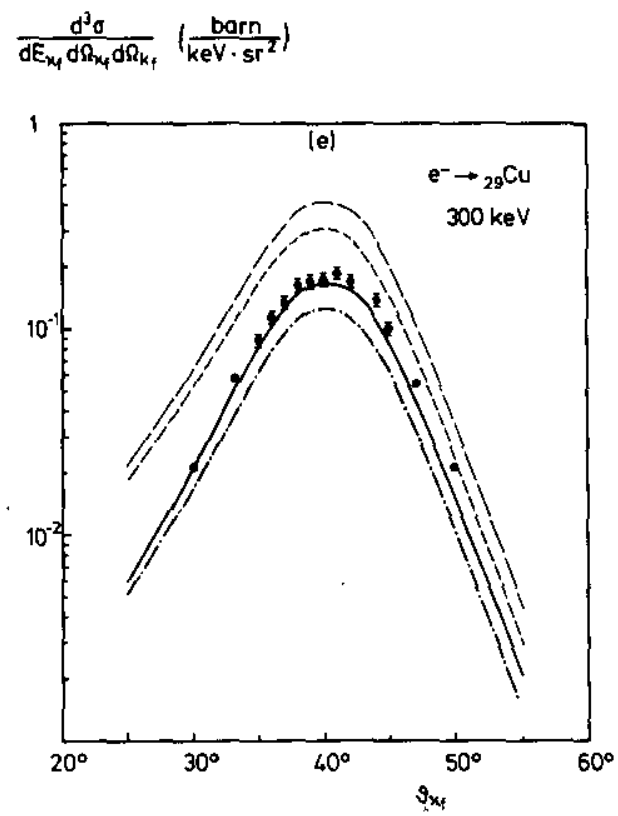

$\frac{d^{3} g}{d E_{x_{1}} d \Omega_{x_{1}} d \Omega_{k_{1}}}\left(\frac{\text { barn }}{\mathrm{keV} \cdot \mathrm{sr}^{2}}\right)$

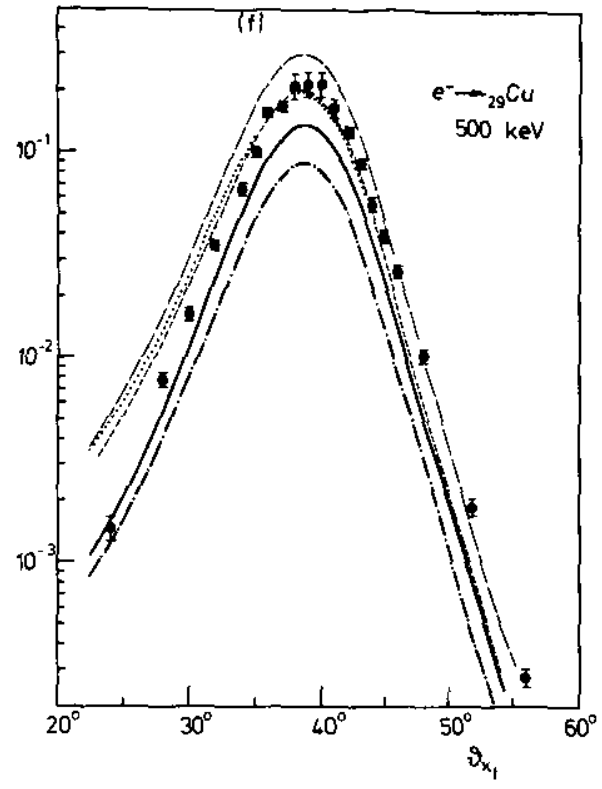

Figure 1. (continued)

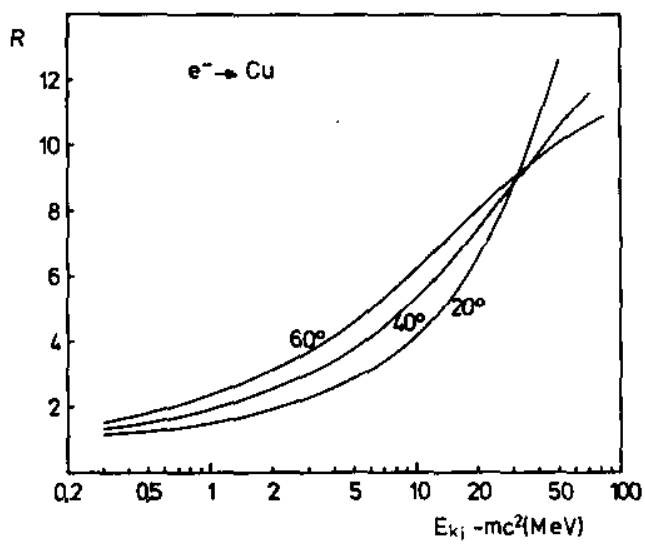

Figure 2. Ratio $R$ between the triply-differential PwBA cross sections for $\mathrm{K}$-shell ionization of $\mathrm{Cu}$, calculated with and without inclusion of spin-fip, respectively, as a function of impact energy $E_{k_{1}}$ for the emission angles $\vartheta_{\kappa_{f}}=20^{\circ}, 40^{\circ}$ and $60^{\circ}$.

where $\gamma=\left(1-\left(Z_{\mathrm{T}} / c\right)^{2}\right)^{1 / 2}$ and $r, \theta, \varphi$ are the spherical coordinates of $r$ with $k_{i}$ as quantization axis. With this function, the target ionization matrix elements $\left\langle\phi_{f}^{\left(\sigma_{f}\right)}|\exp (\mathrm{i} q \boldsymbol{r})| \phi_{i}^{\left(\sigma_{i}\right)}\right\rangle$ and $\left\langle\phi_{f}^{\left(\sigma_{f}\right)}|\exp (\mathrm{i} q \boldsymbol{q}) \boldsymbol{\alpha}| \phi_{i}^{\left(\sigma_{i}\right)}\right\rangle$ can no longer be given in closed form; however, the integral over the azimuthal angle can be evaluated analytically if the reference frame of $\boldsymbol{r}$ is rotated by $-\vartheta_{\kappa_{f}}$ such that the new quantization axis is aligned with $\kappa_{f}$. Transforming $r \cos \theta$ and $r \sin \theta \exp ( \pm \mathrm{i} \varphi)$ occurring in $u_{i}^{\left(\sigma_{i}\right)}$ into the rotated frame and carrying out the spinor multiplications, one is left with a series of two- 
dimensional integrals entering into $W_{s_{f} \sigma_{f} s_{i} \sigma_{i}}^{\mathrm{d}}$

$$
\begin{aligned}
\int \mathrm{d} r \psi_{\kappa_{f}}^{*}(r) \mathrm{e}^{\mathrm{i} \boldsymbol{q} \cdot \boldsymbol{r}} \varphi_{i}(r)\left(\begin{array}{c}
1 \\
x \\
\sqrt{1-x^{2}} \cos \varphi_{r} \\
\sqrt{1-x^{2}} \sin \varphi_{r}
\end{array}\right) \\
=2 \pi N_{\kappa} \int_{0}^{\infty} \mathrm{d} r \int_{-1}^{1} \mathrm{~d} x\left(\begin{array}{c}
J_{0}(y) \\
x J_{0}(y) \\
\mathrm{i} \sqrt{1-x^{2}} J_{1}(y) \\
0
\end{array}\right) r^{\gamma+1} \mathrm{e}^{-z_{\mathbf{r}} r} \\
\quad \times \mathrm{e}^{-\mathrm{i} \kappa_{f} r x}{ }_{1} F_{1}\left(\mathrm{i} \eta_{\kappa}, 1, \mathrm{i} \kappa_{f} r(1+x)\right) \mathrm{e}^{\mathrm{i} q r x \cos \vartheta_{q, \kappa_{j}}}
\end{aligned}
$$

which have been written in vector form in (7). The normalization constant is $N_{\kappa}=$ $\pi^{-2}(\Gamma(2 \gamma+1))^{-1 / 2} 2^{\gamma-5 / 2} Z_{\mathrm{T}}^{\gamma+1 / 2} \exp \left(\pi \eta_{\kappa} / 2\right) \Gamma\left(1-\mathrm{i} \eta_{\kappa}\right)$ with $\eta_{\kappa}=Z_{\mathrm{T}} / \kappa_{f}, x=\cos \vartheta_{r}$ with $\vartheta_{r}, \varphi_{r}$ the angular coordinates of $r$ in the rotated frame, and $y=\operatorname{qr}\left(1-x^{2}\right)^{1 / 2} \sin \vartheta_{q, \kappa_{r}}$. The functions $J_{0}(y)$ and $J_{1}(y)$ are Bessel functions, and ${ }_{1} F_{1}$ is a confluent hypergeometric function (for which it is necessary to use the asymptotic expansion for large arguments $\left.\kappa_{f} r(1+x) \geqslant 20\right)$.

The results of a PWBA calculation with $\phi_{i}^{\left(\sigma_{i}\right)}$ from (6), excluding spin-flip, are also plotted in figure 1. For $\mathrm{Cu}$ (figure $1(f)$ ) and to a somewhat lesser extent for $\mathrm{Ag}$ (figure $1(d)$ ), the difference between the results using a Darwin function or the Dirac function (6) for the K-shell electron, respectively, is rather small. This difference is somewhat greater on the wings of the binary peak where the larger intrinsic momenta of the bound state are enhanced due to the $r^{y}$ contraction of the relativistic wavefunction.

In contrast to the lighter targets, the wavefunction effects are very large for the Au target. From figures $1(a),(b)$ it follows that the use of the relativistic function (6) reduces the cross section by $\sim 30 \%$ near the binary peak maximum. Since the target ionization is described in terms of the same matrix elements both in PWBA and in the Coulomb Born theory, the wavefunction effects are presumably much alike in both theories. Hence, we have derived a correction factor $F_{i}$ as the ratio between the non-flip PWBA results obtained with a Dirac and a Darwin function, respectively, and have multiplied the Coulomb Born spin-flip cross sections by $F_{j}$. As seen in figures $1(a)$, (b) this procedure provides an improved agreement with the data at angles above $30^{\circ}$.

As a next step in investigating wavefunction effects, we have used Darwin functions for both states, $\phi_{f}^{\left(\sigma_{f}\right)}$ and $\phi_{i}^{\left(\sigma_{i}\right)}$, of the secondary electron, as has been done in the PWBA calculations given in Bonfert et al (1991) or Jakubassa-Amundsen (1989). The effect of describing $\phi_{f}^{\left(\sigma_{f}\right)}$ by a Darwin function is readily seen in the PWBA results of Walters et al (1991) where this theory is compared with the one using Coulomb waves for $\phi_{f}^{\left(\sigma_{f}\right)}$ like in (2): While both theories nearly coincide for $\mathrm{Cu}$ and $\mathrm{Ag}$, the cross sections are substantially reduced for the Au target when the improved (Darwin) function is used. For the sake of completeness, we have included the non-flip PwBA results with Darwin functions for $\phi_{i}^{\left(\sigma_{1}\right)}$ and $\phi_{f}^{\left(\sigma_{f}\right)}$ in figures $1(a),(b)$. Assuming tentatively that a similar reduction occurs when each of the states $\dot{\psi}_{f}^{\left(s_{i}\right)}$ and $\dot{\psi}_{i}^{\left(s_{1}\right)}$ of the primary electron is replaced by a Darwin function, and applying this estimate to the Coulomb Born theory, one expects to get a total reduction of about a factor of 2 at the smaller angles. This would bring the Au results to a reasonable agreement with the data for $\vartheta_{\kappa f} \geqslant 20^{\circ}$. 
So far, only the decrease of the Au data for angles below $20^{\circ}$ remains unexplained, where theory provides increasing cross sections. In this context it should be noted that the first-order treatment of the electron-electron coupling in (2) breaks down for $\vartheta_{\kappa_{f}} \rightarrow 0$, because the two outgoing electrons will acquire the same momenta, $\boldsymbol{\kappa}_{f} \rightarrow \boldsymbol{k}_{f}$, with the result of a strong interelectronic repulsion. Brauner et al (1989) have considered the electron-electron interaction non-perturbatively by replacing the two-electron final state $\left|\psi_{f}^{\left(s_{f}\right)} \phi_{f}^{\left(\sigma_{f}\right)}\right\rangle$ by a distorted wave $\left|\psi_{f}^{\left(s_{f}\right)} \phi_{f}^{\left(\sigma_{f}\right)} \chi_{f}\right\rangle$ with $\chi_{f}=$ $\exp \left(\pi \eta_{e e} / 2\right) \Gamma\left(1+\mathrm{i} \eta_{e e}\right){ }_{1} F_{1}\left(-\mathrm{i} \eta_{e e}, 1,-\mathrm{i}\left(k_{e c} r+k_{e e} r\right)\right)$ where $\eta_{e e}=-1 /\left(2 k_{e e}\right), r$ is the interelectronic coordinate and $\boldsymbol{k}_{\mathrm{ee}}=\left(\boldsymbol{\kappa}_{f}-\boldsymbol{k}_{f}\right) / 2$ the relative momentum of the two outgoing electrons. Similar distorted-wave prescriptions are often simplified by using peaking approximations which reduce the effect of distortion simply to the normalization constant of $\chi_{f}$ (Garibotti and Miraglia 1980). Following this idea, we estimate the reduction of the $(\mathrm{e}, 2 \mathrm{e})$ cross section by the interelectronic repulsion by scaling

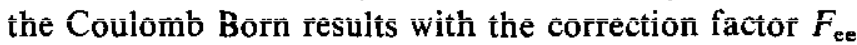

$$
F_{\mathrm{ee}}=\mathrm{e}^{\pi \eta_{\mathrm{ee}}}\left|\Gamma\left(1+\mathrm{i} \eta_{\mathrm{ee}}\right)\right|^{2}=\frac{2 \pi\left|\eta_{\mathrm{ee}}\right|}{\mathrm{e}^{2 \pi \mid \eta_{\mathrm{ec}}}-1} .
$$

For $300 \mathrm{keV}$ electron impact where the effect of $F_{\mathrm{ee}}$ is largest, one finds only a rather small reduction of the cross section $\left(\leqslant 10 \%\right.$ ) at angles above $10^{\circ}$ (figure $1(a)$ ), because the electronic momenta and hence $k_{\text {ee }}$ are very lärge.

In conclusion, we have performed calculations of triply-differential electron emission cross sections in the Coulomb Born approximation, varying both impact velocity and target charge. We have found that this theory, when spin-flip is included, provides a reasonable explanation of the binary-peak data for the two lighter targets $(\mathrm{Cu}$ and $\mathrm{Ag}$ ). Large discrepancies persist, however, for the heaviest $\mathrm{Au}$ ) target, particularly at small electron angles. The consideration of the electron-electron repulsion in an approximate non-perturbative way improves only marginally the agreement with experiment. The use of a more accurate (relativistic) wavefunction for the bound electron or for a continuum electron has a considerable effect on the cross section for the Au target, while leading only to small corrections for $\mathrm{Cu}$ or $\mathrm{Ag}$. Clearly, the use of exact relativistic wavefunctions for all electronic states is required in order to give a better accord with the data on electron ejection from very heavy targets.

\section{Acknowledgment}

I am very grateful to $\mathrm{W}$ Nakel for stimulating this work and for keeping me informed on his experiments prior to publication. I should also like to thank $H$ Ast for helpful discussions and the GSI Darmstadt for financial support.

\section{References}

Bjorken D and Drell S D 1964 Relativistische Quantenmechanik (Mannheim: Bibliographisches Institut) Bonfert J, Graf H and Nakel W 1991 J. Phys. B: At. Mol. Opt. Phys. 241423

Brauner M, Briggs J S and Klar H 1989 J. Phys. B: At. Mol. Opt. Phys. 222265

Das J N 1972 Nuovo Cimento 12B 197

Davidović D M, Moiseiwitsch B L and Norrington P H 1978 J. Phys. B: At. Mol. Phys. 11847

Ehrhardt H, Jung K, Knoth G and Schlemmer P 1986 Z. Phys. D 13

Garibotti C R and Miraglia J E 1980 Phys. Rev. A 21572 
Jakubassa-Amundsen D H 1989 Z. Phys. D 11305

Lahmam-Bennani A 1991 J. Phys. B: At. Mol. Opt. Phys. 242401

Madison D H, Calhoun R V and Shelton W N 1977 Phys. Rev. A 16552

McCarthy I E and Weigold E 1976 Phys. Rep. C 27275

Moiseiwitsch B L 1980 Adv. At. Mol. Phys. 16281

Møller C 1932 Ann. Phys., Lpz. 14531

Pindzola M S and Buie M J 1988 Phys. Rev. A 373232

Ruoff H and Nakel W 1987 J. Phys. B: At. Mol. Phys. 202299

Schüle E and Nakel W 1982 J. Phys. B: At. Mol. Phys. 15 L639

Walters H R T, Ast H, Whelan C T, Dreizler R M, Graf H, Schröter C D, Bonfert J and Nakel W $1991 Z$. Phys. D to appear 